\title{
Research on Hidden Failure Reliability Modeling of Electric Power System Protection
}

\author{
Jingjing Zhang, Ming Ding, Xianjun Qi, Yi Guo \\ School of electrical engineering and automation, Hefei University of Technology, Hefei, China \\ Email: dragonzjj@126.com
}

Received February, 2013

\begin{abstract}
Aiming at digital relay protection system, a novel hidden failure Markov reliability model is presented for a single main protection and double main protection systems according to hidden failure and protection function under Condition-Based Maintenance (CBM) circumstance and reliability indices such as probability of protection system hidden failure state are calculated. Impacts of different parameters (containing impacts of human errors) to hidden failure state probability and the optimal measures to improve reliability by variable parameter method are also analyzed. It's demonstrated here that: Compared to a single main protection, double main protection system has an increased hidden failure probability, thus the real good state probability decreases, two main protections' reliability must be improved at the same time, so configuration of the whole protection system for the component being protected can't be complicated. Through improving means of on-line self-checking and monitoring system in digital protection system and human reliability, the real application of CBM can decrease hidden failure state probability. Only through this way can we assure that the protection systems work in good state. It has a certain reference value to protection system reliability engineering.
\end{abstract}

Keywords: Double Main Protection System; Hidden Failure; Markov Method; Condition-Based Maintenance (CBM); Human Error

\section{Introduction}

Ref.[1-5] are the first to explore hidden failures in protection system carefully, later many experts carried research on protection hidden failure and its contribution to protection system reliability and power system reliability and have obtained many good results[6-14]. Now, CBM (Condition-Based Maintenance) is presented to apply in power system and protection system in China, hidden failure of protection is defined as a function defect of protection device before; under new CBM circumstance $[15,16]$, hidden failure is defined as a hidden defect of protection that can't be detected by means of CBM such as on-line self-checking and monitoring system, and it may result in mal-operation or non-operation of protection system under certain condition, for example, settings of protection don't change according to the operation mode of protected equipment. Application of CBM is based on condition of protection device instead of operation time, it can decrease test time and test cost. CBM is carried on aiming at hidden failure state of protection system; the level of its putting into practice determines the level of protection system's good state.

When carrying on reliability research of protection system using Markov method, it's often assumed that failure rate and repair rate of protection is constant, and CBM Substitutes routine test by using on-line self-checking and monitoring method, the routine test interval doesn't need to be considered. In the following, aiming at digital relay protection system, a novel hidden failure Markov reliability model will be presented for a single main protection and double main protection system separately, according to hidden failure and protection function under CBM circumstance, reliability indices such as probability of protection system hidden failure state will be calculated. Impacts of different parameters (containing impacts of human errors) to hidden failure state probability and the optimal measures to improve reliability by variable parameter method will be analyzed. It can present a certain reference value to protection system reliability engineering and application of CBM in protection system.

\section{Hidden Failure Reliability Model of Single Protection System}

First, hidden failure reliability model of a single main protection is presented by Model 1, as Figure 1 shows. 
The protected component has two states: normal state UP and outage state DN; protection has four states: normal state UP and failure state DN, hidden non-operation state DUN and hidden mal-operation state DUM. It's assumed that CBM can't check all failures of protection system, so protection system may stay in hidden failure state; because hidden failure state isn't failure state, it has no fault consequence, it doesn't belong to mal-operation state or non-operation state; it only shows that the protection system is in a hidden unhealthy state and may malfunction under some circumstances. For example, protection system in hidden failure state may incorrectly mal-operate when fault happens outside the protected zone, it may incorrectly refuse to operate when fault happens inside the protected zone.

When doing research on reliability of protection system, each state of the system must be considered, so is probability of each state and the transition rate between states. Markov process is a useful tool to analyze these questions. In Figure 1, state 1 is normal state of component being protected and protection equipment; state 2 is that when component fails, its protection operates correctly; after component being repaired, it goes to state 1 ; state 3 is that component is good, protection has selfcheckable failure; state 4 is that component is good, protection has non-self-checkable mal-operation failure; state 5 is that component is good, protection has nonself-checkable non-operation failure; state 6 is that hidden mal-operation is triggered under external fault or it's own fault condition, and non-self-checkable mal-operation of protection happens; state 7 is that when component fails, non-self-checkable non-operation of protection happens; if component is repaired first, it goes to state 3 ; if protection is repaired first, it goes to state 2; state 8 is that component fails, protection's mal-operation is considered as correct operation, after component is repaired, it goes to state 4 . Hidden mal-operation state (state 4) can convert to hidden non-operation state (state 5) and vice versa.

In Figure 1, $\lambda_{C}$ is failure rate of component being protected, $\mu_{c}$ is repair rate of component being protected, $\lambda_{P}$ is failure rate of protection(it consists of hardware failure rate and software failure rate), $C_{1}$ is self-checkable success rate of protection, $C_{3}$ is mal-operation percentage of protection, $C_{5}=C_{3} \lambda_{P}\left(1-C_{1}\right)$ is non-selfcheckable mal-operation rate of protection, $C_{6}=$ $\left(1-C_{3}\right) \lambda_{P}\left(1-C_{1}\right)$ is non-self-checkable non-operation rate of protection, $\mu_{1}$ is repair rate of protection, $\lambda_{\text {ext }}$ is failure rate of external fault of component being protected.

$$
\begin{aligned}
& P(n) \times \mathbf{B}=0 \\
& \sum_{i=1}^{8} p_{i}=1
\end{aligned}
$$

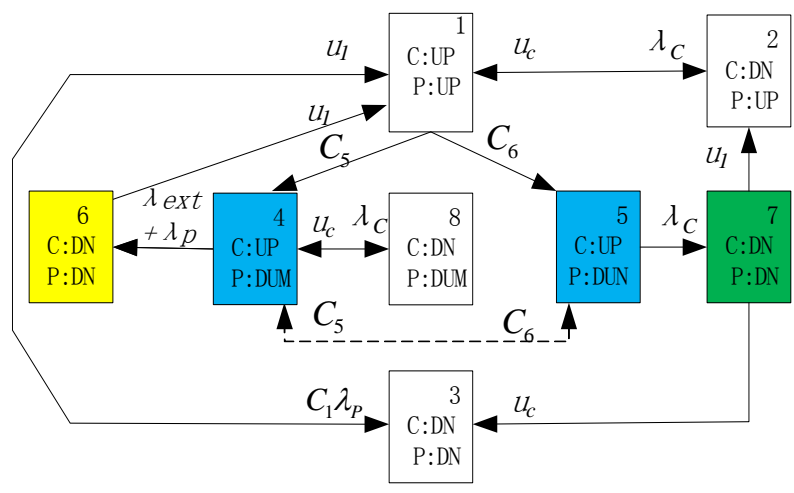

Figure 1. Hidden failure reliability model of single main protection system.

Through Equation (1) and (2), we can get stable state transition probability matrix $B$ and each state probability $P(n)=\left[p_{1}, p_{2}, \cdots, p_{8}\right]$.

Defining hidden failure state probability of protection

$$
p_{\text {hidden }}=p_{4}+p_{5}
$$

Defining hidden mal-operation failure state probability of protection

$$
p_{h w}=p_{4}
$$

Defining hidden non-operation failure state probability of protection

$$
p_{h j}=p_{5}
$$

\section{Hidden Failure Reliability Model of Double Main Protection System}

Reliability model of double main protection system is presented by Model 2, as Figure 2 shows. The model is similar to Model 1, but it's more complicated for double main protection, protection $\mathrm{P} 1$ and $\mathrm{P} 2$ has identical position. Define $\lambda_{P}$ as failure rate of protection $\mathrm{P} 1$, the parameters of main protection $\mathrm{P} 1$ is identical to that of Model 1.

As for protection P2, $\lambda_{P 2}$ is failure rate of protection, $C_{2}$ is self-checkable success rate of protection, $C_{4}$ is mal-operation percentage of protection, $C_{7}=C_{4} \lambda_{P 2}\left(1-C_{2}\right)$ is non-self-checkable mal-operation rate of protection, $C_{8}=\left(1-C_{4}\right) \lambda_{P 2}\left(1-C_{2}\right)$ is non-self-checkable non-operation rate of protection, $\mu_{2}$ is repair rate of protection, $\mu$ is repair rate of both protection at the same time. Define: $C_{9}=C_{1} \lambda_{P}, \quad C_{10}=C_{2} \lambda_{P 2}$.

Defining reliability indices similar to Model 1 ,

$$
\begin{gathered}
p_{\text {hidden }}=p_{5}+p_{6}+p_{7}+p_{8}+p_{9}+p_{10}+p_{11}+ \\
p_{12}+p_{13}+p_{14}+p_{15}+p_{16} \\
p_{h w}=p_{5}+p_{6}+p_{7}+p_{8}+p_{9}+p_{10}+p_{14} \\
p_{h j}=p_{13}+p_{15}+p_{16}
\end{gathered}
$$




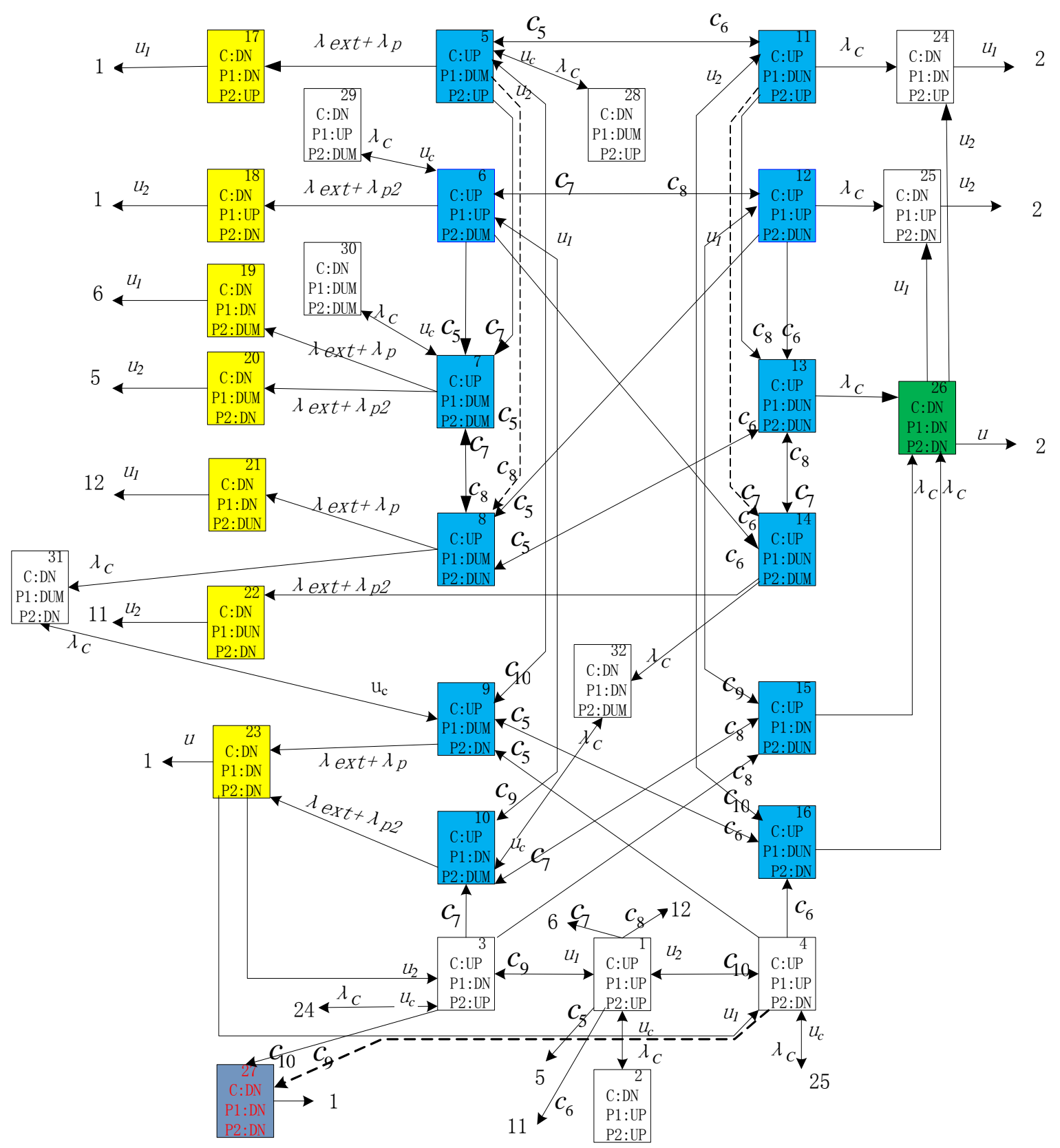

Figure 2. Hidden failure reliability model of double main protection system.

\section{Hidden Failure Reliability Model of Single Protection System Considering Human} Error

Human error can be defined as any improper action, resulting in events that will affect the proper action of the system. From a system point of view, with reliable hardware and software, human error remains as a great threat to system safety [17-20]. For example, incorrect operation of operating personnel occurred in South America and North Mexico interconnected power grid cascading outage on Sept. 8, 2011, so now it has been an important factor that deserves our attention.

The reasons for human errors are fatigue and sleeplessness, anger, emotional upsets, lack of skill, hunger, letdown from low blood sugar, medication, drugs and so on. Human error can be divided into seven kinds: design error, operator error, fabrication error, maintenance error, contributory error, inspection error and handling error.

There are numerous techniques available for conducting human reliability assessment, such as THERP (technique for human error rate prediction), HEART(human error assessment and reduction technique) and so on. Through these methods we can achieve the failure prob- 
ability of human operation. Here human error is described by a mean failure probability of a constant.

The two fault modes for protection system are maloperation and non-operation, the impact of human error to protection system also has two kinds: mal-operation and non-operation. In the following analysis, it's assumed that human error appears after some operation and repair.

Hidden failure reliability model of single main protection system considering human error is presented by Model 3, as Figure 3 shows. This model is based on Model 1, two kinds of human errors are considered: 1) protection system mal-operation owing to incorrectly operation of operating personnel, for example, dispatching personnel or operator on duty fails to follow correct procedure; 2) protection system are not completely good after repair, for example, settings of protection don't change after repair, this may cause hidden mal-operation or non-operation of protection system.

In Figure 3, when protection P trips incorrectly owing to human error, state 1 goes to state 6; when protection $\mathrm{P}$ is not repaired completely owing to human error, state 3 goes to state 4 (hidden mal-operation state) or state 5 (hidden non-operation state). As for protection $\mathrm{P}, K_{\mathrm{h} 1}$ is a mean human error rate; $v_{1}$ is mal-operation percentage owing to human error; so we can achieve the reliability indices that are identical to Model 1.

\section{Case Studies}

Here, take the data of Table $\mathbf{1}$ for example, we calculate the reliability indices of the three models and analyze the results; the computation results are shown as Table 2. Using variable parameter method, $p_{\text {hidden }}$ curve of Model 1 under different $C_{1}$ is shown as Figure 4 (that is to say, under certain $C_{1}$, when $\lambda_{P}$ increases, we can obtain the curve of $p_{\text {hidden }}$ ), $p_{\text {hidden }}$ curve of Model 2 under different $C_{1}$ is shown as Figure 5 (to Model 2, when $\lambda_{P 2}$ increases, $p_{\text {hidden }}$ curve under different $C_{2}$ is the same as Figure 5), impact of human error to $p_{\text {hidden }}$ of Model 3 is shown as Figure 6.

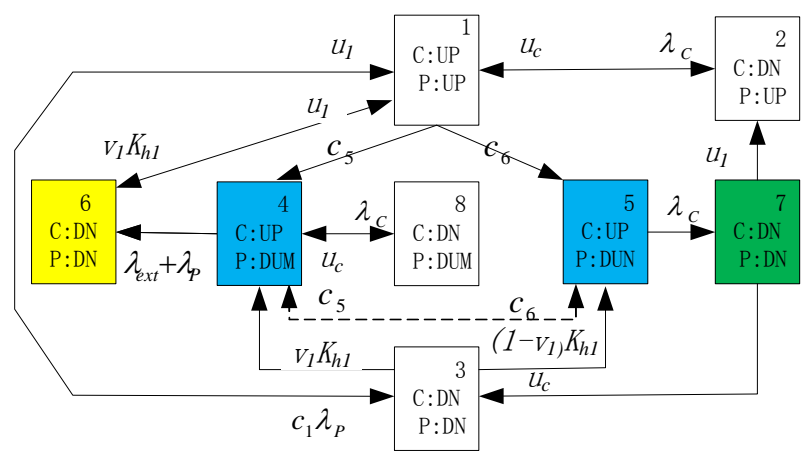

Figure 3. Hidden failure reliability model of single main protection system considering human error.
Table 1. Reliability base data for the computation.

\begin{tabular}{cccc}
\hline Parameter & value & Parameter & value \\
\hline$\lambda_{C} / \mathrm{y}^{-1}$ & 0.04 & $\mu_{C} / \mathrm{h}^{-1}$ & 0.25 \\
$\lambda_{P} / \mathrm{y}^{-1}$ & 0.08 & $\mu_{1} / \mathrm{h}^{-1}$ & 0.25 \\
$\lambda_{P 2} / \mathrm{y}^{-1}$ & 0.08 & $\mu_{2} / \mathrm{h}^{-1}$ & 0.25 \\
$\nu_{1}=c_{3}=c_{4}$ & 0.5 & $\mu / \mathrm{h}^{-1}$ & 0.25 \\
$\lambda_{\text {ext }} / \mathrm{y}^{-1}$ & 0.005 & $K_{h 1} / \mathrm{y}^{-1}$ & 0.001 \\
$C_{1}=C_{2}$ & 0.9 & & \\
\hline
\end{tabular}

Table 2. Reliability index calculation results.

\begin{tabular}{cccc}
\hline \multirow{2}{*}{ Model } & \multicolumn{3}{c}{ Reliability index } \\
\cline { 2 - 4 } & $p_{\text {hidden }}$ & $p_{h w}$ & $p_{h j}$ \\
\hline Model 1 & 0.1263 & 0.0430 & 0.0833 \\
Model 2 & 0.2318 & 0.0842 & 0.0119 \\
Model 3 & 0.1263 & 0.0430 & 0.0833 \\
\hline
\end{tabular}

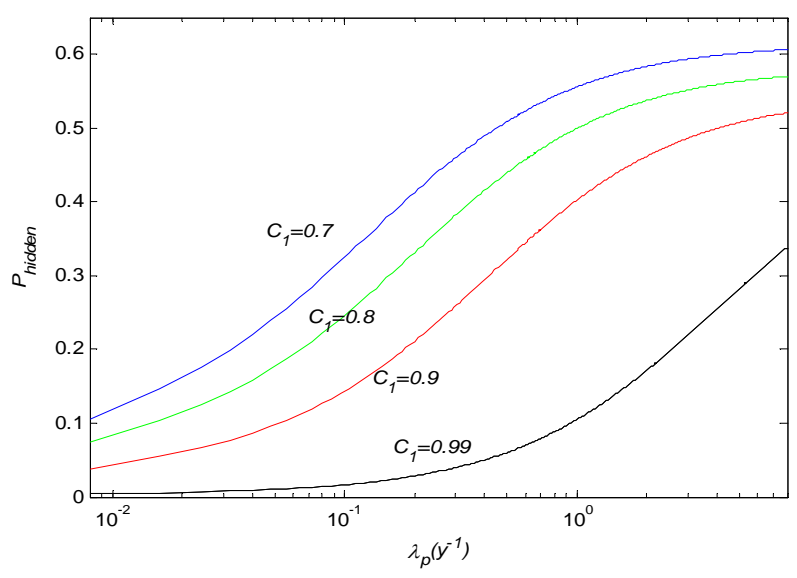

Figure 4. $p_{\text {hidden }}$ curve of Model 1 under different $C_{1}$.

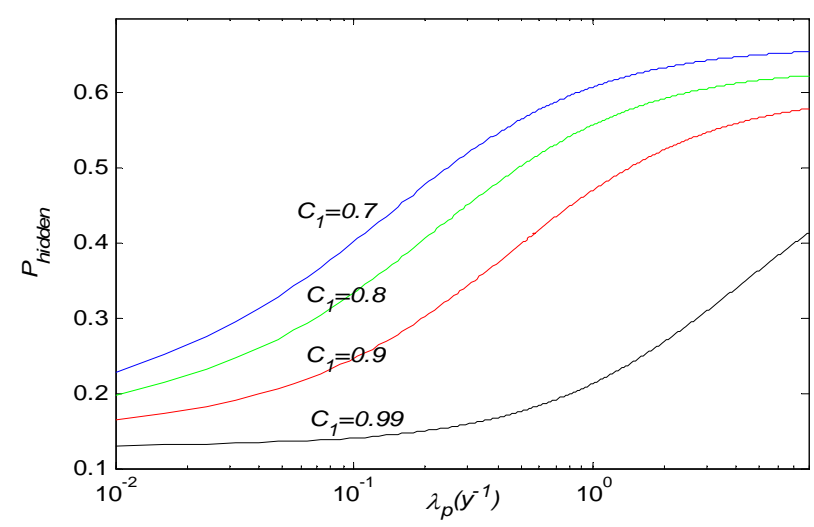

Figure 5. $p_{\text {hidden }}$ curve of Model 2 under different $C_{1}$. 


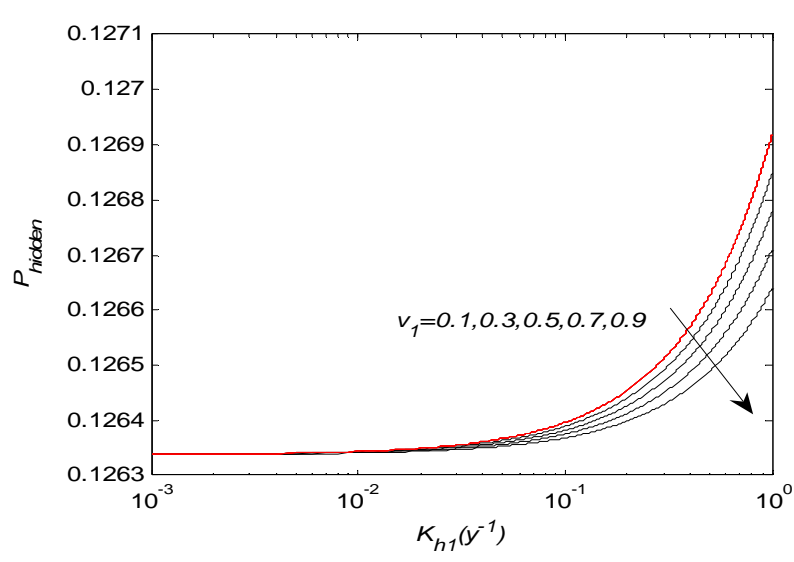

Figure 6. Impact of human error to $p_{\text {hidden }}$ of Model 3.

From Table 2, Figure 4 to Figure 6, we can draw the conclusions:

- $\quad$ Compared to Model 1, Model 2 has a higher $p_{\text {hidden }}$ and $p_{h w}$, a lower $p_{h j}$, this shows that redundant protection can decrease hidden non-operation state probability, but at the same time it increases hidden mal-operation state probability, thus hidden failure state probability increases, so the completely good state probability of protection system decreases. When using redundant protection, we must consider it.

- $\quad$ To Model 3, when $K_{h 1}$ increases, $p_{\text {hidden }}$ increases; when $\mathrm{v}_{1}$ increases as the arrow shows, $p_{\text {hidden }}$ decreases; compared with Model 1 , when $K_{h 1}$ is small, it rarely has impact on these indices. This means that mean human error rate and mal-operation percentage owing to human error can affect hidden failure state probability, so we must take all measures that can be done to decrease human rate error and improve reliability of protection system.

- $\quad$ From Figure 4 and Figure 5, we can see that the curves of hidden failure state probability of Model 1 and Model 2 under different $C_{1}$ are similar; when $\lambda_{P}$ increases, $p_{\text {hidden }}$ increases; when $C_{1}$ increases, $p_{\text {hidden }}$ decreases. This shows that failure rate of protection and self-checkable success rate of protection can affect reliability of protection system greatly, and two main protection's reliability must be improved at the same time. Through improving means of on-line self-checking and monitoring system in digital protection system, the real application of CBM can decrease hidden failure state probability. When reliability of single main protection system is high, we can consider simplified configuration of the whole protection system.

\section{Conclusions}

Aiming at digital protection system, we must take meas- ures not only to decrease mal-operation probability and non-operation probability, but also to decrease hidden failure state probability. Compared to a single protection, double main protection system has an increased hidden failure state probability, thus the real good state probability decreases, two main protection's reliability must be improved at the same time, so configuration of protection system for the component being protected can't be complicated(such as two out of three vote). Human error rate can increase hidden failure state probability of protection system, human error must be reduced during normal operation and maintenance process. Through improving means of on-line self-checking and monitoring system in digital protection system, the real application of CBM can decrease hidden failure state probability. Only through this way can we assure that the protection systems work in good state. It has a certain reference value to protection system reliability engineering.

\section{Acknowledgements}

This project is supported by State Grid Corporation of China Major Projects on Planning and Operation Control of Large Scale Grid (SGCC-MPLG024 -2012 ), the National Natural Science Foundation of China under Grant (51007017) and Specialized Research Fund for the Doctoral Program of Hefei University of Technology

(2012HGBZ0657), the author thanks.

\section{REFERENCES}

[1] P. M. Anderson and S. K. Agarwal, "An Improved Model for Protective-system Reliability,” IEEE Transactions on Relibility, Vol. 41, No. 3, 1992, pp. 422-426. doi:10.1109/24.159812

[2] S. Tamronglak, “Analysis of Power System Disturbances Due to Relay Hidden Failures,” Ph.D. dissertation, Virginia Polytechnic State University, Blacksburg, 1994.

[3] S. Tamronglak, S. H. Horowitz, A. G. Phadke and J. S. Thorp, "Anatomy of Power System Blackouts: Preventive relaying Strategies," IEEE Transactions on Power Delivery, Vol. 11, No. 2, 1996, pp. 708-715. doi:10.1109/61.489327

[4] A. G. Phadke and J. S. Thorp, "Expose Hidden Failures to Prevent Cascading Outages,” IEEE Computer Applications in Power, Vol. 9, No. 3, 1996, pp. 20-23. doi:10.1109/67.526849

[5] P. M. Anderson, G. M. Chintaluri, S. M. Magbuhat and R. F. Ghajar, "An Improved Reliability Model for Redundant Protective Systems-Markov Models," IEEE Transactions on Power System, Vol. 12, No. 2, 1997, pp. 573-578. doi:10.1109/59.589606

[6] R. Billinton, M. Fotuhi-Firuzabad and T. S. Sidhu, "Determination of the Optimum Routine Test and Self-checking Intervals in Protective Relaying Using a Reliability Model,” IEEE Transactions on Power System, 
Vol. 17, No. 3, 2002, pp. 663-669.

doi:10.1109/TPWRS.2002.800871

[7] D. C. Elizondo, "A Methodology to Assess and Rank the Effects of Hidden Failures in Protection Schemes based on Regions of Vulnerability and Index of Severity,” Ph.D. dissertation, Virginia Polytechnic and State University, Blacksburg, Virginia, April 2003.

[8] X. F. Xiao, O. Y. F. Qian, Z. Q. Jia, et al., "Probabilistic Model for the Relay Protection System's Correct Failure Removal," Automation of Electric Power Systems, Vol. 31,No. 7, 2007,pp. 12-14.

[9] S. S. Fu and W. H. Xiong, “A New Method for Reliability Analysis of Protection in Power Systems," Automation of Electric Power Systems, Vol. 30, No. 16, 2006, pp. 32-35.

[10] Z. S. Xue, W. Chao and C. D. Xiao, "Reliability Analysis Model for Protective Relaying System of UHV Power Network Based on Markov State-Space Method," Power System Technology, Vol. 33,No. 13,2008, pp. 94-99.

[11] Z. J. Jing and D. Ming, "Summary of Research on Hidden Failures in Protection Systems," Proceedings of International Conference on Electrical Machines and Systems, Vol. 10, 2008, pp. 870-872.

[12] Z. Tao, W. Fang and J. Naizheng. “A Novel Algorithm of Determining the Optimal Routine Test Interval of the Dual-redundant Relay Protection System," Automation of Electric Power Systems, Vol. 34, No. 10, 2010, pp. 67-70.

[13] A. H. Etemadi and M. Fotuhi-Firuzabad, "New Considerations in Modern Protection System Quantitative Reliability Assessment," IEEE Transactions on Power Deliv- ery, Vol. 24, No. 4, 2010, pp. 2213-2222. doi:10.1109/TPWRD.2010.2051463

[14] A. H. Etemadi and M. Fotuhi-Firuzabad, "Quantitative Assessment of Protection System Reliability Incorporating Human Errors,” Part O:J.Risk and Reliability, Vol. 222, 2008, pp. 255-263.

[15] G. Xiang, “Application Technology of Condition Based Maintenance of Relay Protection,” China Electric Power Press, 2008.

[16] G. Xiang and L. Shaojun. "Condition Maintenance and Implementation of Relay Protection,” Relay, Vol. 33, No. 2, 2005, pp. 23-27.

[17] D. O. Koval and H. L. Floyd, "Human Element Factors Affecting Reliability and Safety," IEEE Transactions on Industry Applications, Vol. 34, No. 2, 1998, pp. 406-414. doi:10.1109/28.663487

[18] Z. Bingquan and F. Xiang, "Experiment Research on Cognition Reliability Model of Nuclear Power Plant," Journal of Tsinghua University, Vol. 39, No. 5, 1999, pp. 122-125.

[19] Arizona Public Service(APS), "Cause of Widespread Outage under Investigation[EB/OL],”[2011-9-9]. http://www.aps.com.

[20] M. Anjia, Z. Geli and L. Yuechun, "Analysis on Large-scale Blackout Occurred in South America and North Mexico Interconnected Power Grid on Sept. 8, 2011 and Lessons for Electric Power Dispatching in China,” Power System Technology, Vol. 36, No. 4, 2012, pp. 74-78. 